\title{
¿AGRICULTURA DESARROLLADA O SIMPLEMENTE COMERCIAL? EL CASO VALENCIANO
}

\author{
Joaquín Farinós Dasí*
}

\section{RESUMEN}

¿Realmente se puede considerar a la agricultura valenciana como innovadora y modernizada? La mala situación por la que está atravesando el sector y la progresiva pérdida de rentabilidades, desde hace varias décadas con un ritmo decreciente mayor que la media nacional pese a partir de una situación más ventajosa, llevó a plantear un estudio que nos acercara a la realidad tecnológica de nuestra agricultura. Los resultados arrojan una agricultura sucedánea de la modernidad, donde predomina el comportamiento rápidamente imitativo.

Con fuerte tradición cultural, tradicionalmente volcada a los mercados urbanos, locales, regionales e incluso internacionales, y con un confuso, aunque al menos constatado, sentido económico-contable, los resultados de la investigación la ubican en un escalón 'comercial' cada vez más saturado por la competencia. Se detectan importantes deficiencias tanto para poder hacer frente a la competencia derivada de la reciente o inminente llegada de otras agriculturas, especialmente desde países menos desarrollados que gozan de grandes ventajas en el precio de la mano de obra, como para poder reubicarse en un escalofón superior entre otras agriculturas más desarrolladas como la americana, holandesa o la israelí incluso.

Los motivos los encontramos especialmente en la calidad del capital humano, a todos los niveles. La incapacidad organizativa y de gestión, obvia para la inmensa mayoría de los propios agricultores dada su 'anómala' formación, alcanza también al continuo investigación-transferencia-difusión del cual ellos sólo son, lamentablemente, los beneficiarios finales. Finalmente, el panorama se completa con otra importante limitación: la minidimensión de las explotaciones, problemática en perfecta sintonía con la citada anteriormente, con la que llega a constituir una viciada espiral que es necesario romper.

\begin{abstract}
Is valencian agriculture really innovative and modern? Bad situation it is going through, progressively loosing profitabilities, more than national mean, takes us to study its technological reality. Outcome presents an ersatz of modernity agriculture, with prevalence of fastly imitative performances.
\end{abstract}

* Profesor del Departamento de Geografía de la Universidad de Valencia. 
Traditionaly related with urban markets, locals, regionals or internationals, owner of an important cultural tradition and with a slight economic sense, investigation results show us an agriculture in 'commercial' stage, hounded by competence, with difficulties to defende against less developed countries agricultures and incapacitated for be between best world agricultures (U.S.A., Holland, even Israel).

Reasons are in human capital quality, in all levels. Management and organizational incapacities, not only in farmers but in segments related with research-transferencediffusion activities, are a lot extended; all this in a country of little sizes, too much small rural holdings.

\section{Introducción}

El contenido de este artículo es el resultado de una investigación cuyo objeto de estudio fue la forma en que se producen los procesos de innovación y difusión tecnológica dentro de la agricultura valenciana. Se trataba de averiguar cuáles son los mecanismos mediante los que se crean y difunden las innovaciones y cuáles son los principales estrangulamientos a los que se enfrentan.

La justificación de abordar un tema como éste viene dada por la importancia que la modernización y el cambio técnico tienen para el sector de cara a la competencia creciente de las nuevas agriculturas comerciales del sur mediterráneo y de las más desarrolladas del norte. La posibilidad de cambio y mejora técnica afectará al grado de competitividad, a la posibilidad de generar riqueza, en consecuencia al incremento de las rentas y en última instancia al propio desarrollo regional.

La agricultura es un sector con un fuerte peso específico dentro de la economía valenciana, y uno de los que goza de las condiciones más favorables para ser competitivo. Dispone tanto de los recursos naturales necesarios como, potencialmente, de una capacidad, «know-how» o «savoir faire» práctico, productivo y comercial, que permiten presuponerle una serie de ventajas que otros sectores económicos no tienen.

No se trata de darle una mayor importancia de la que para la economía regional tiene, pero sí de cuidar por el adecuado aprovechamiento de las potencialidades endógenas, de entre las cuales la agraria es una, ciertamente significativa, con la que se ha contado para el proceso de industrialización y desarrollo económico, tanto autonómico como nacional. Recuérdese la idea de la complementariedad productiva de las regiones españolas a la que se refería PERPIÑÁ o la escuela de autores como José Manuel NAREDO, que se ocuparon minuciosamente de explicar el papel que ha jugado la agricultura en el desarrollo económico, industrial o capitalista español ${ }^{1}$.

Es cierto, no obstante, que este flujo de recursos no ha sido únicamente en este sentido, es decir de la agricultura a la industria, sino que también se da en sentido inverso. Es más, en el caso de la agricultura valenciana más productiva predomina desde fechas históricas, pero sobre todo desde el proceso de industrialización y urbanización ligado a ella, la corriente que desde otros sectores económicos llega a la agricultura ${ }^{2}$.

1 Perpiñá Grau, R. (1972): «De Economía Hispana», y Naredo, J. M.; Leal, J. L.; Leguina, J. y Tarrafeta, L. (1975): «La agricultura en el desarrollo capitalista español (1940-1970)».

2 Estudios como el de Burriel de Orueta, E. L. (1971): «La Huerta de Valencia, sector sur. Estudio de Geografía Agraria» constatan el papel del capital urbano como remodelador y modernizador del campo valenciano desde fechas históricas (finales del XIX, principios del XX). Desde una perspectiva más reciente, Eladio Arnalte Alegre se ha constituido como uno de los más importantes teóricos de una nueva forma de agricultura ligada a ese proceso de industrialización-urbanización, especialmente desde los años 60s, que es la agricultura a tiempo parcial, extraordinariamente extendida en nuestro territorio. (Arnalte, E. (1980): «La agricultura a tiempo parcial en el País Valenciano»). 


\section{Metodología}

Para delimitar conceptualmente el contenido de la investigación y establecer las bases de partida, en el método científico hipótesis, para su posterior desarrollo, es necesaria una previa revisión teórica.

Respecto a la primera cuestión, la delimitación conceptual, el siguiente esquema sintetiza los conceptos básicos, diferenciando tecnología de técnica, y en consecuencia cambio tecnológico del técnico. Del mismo modo establece la relación entre uno y otro, así como reproduce el continuo que, a nivel teórico, configuran las tres etapas consecutivas que constituyen el cambio técnico.

En cuanto a la segunda, el establecimiento de hipótesis de trabajo, la intención fue la de plantear un análisis global de la difusión. Se trataba de integrar tanto el tradicional enfoque de la demanda, empleado mayormente desde la Sociología Rural, con el más novedoso de la oferta.

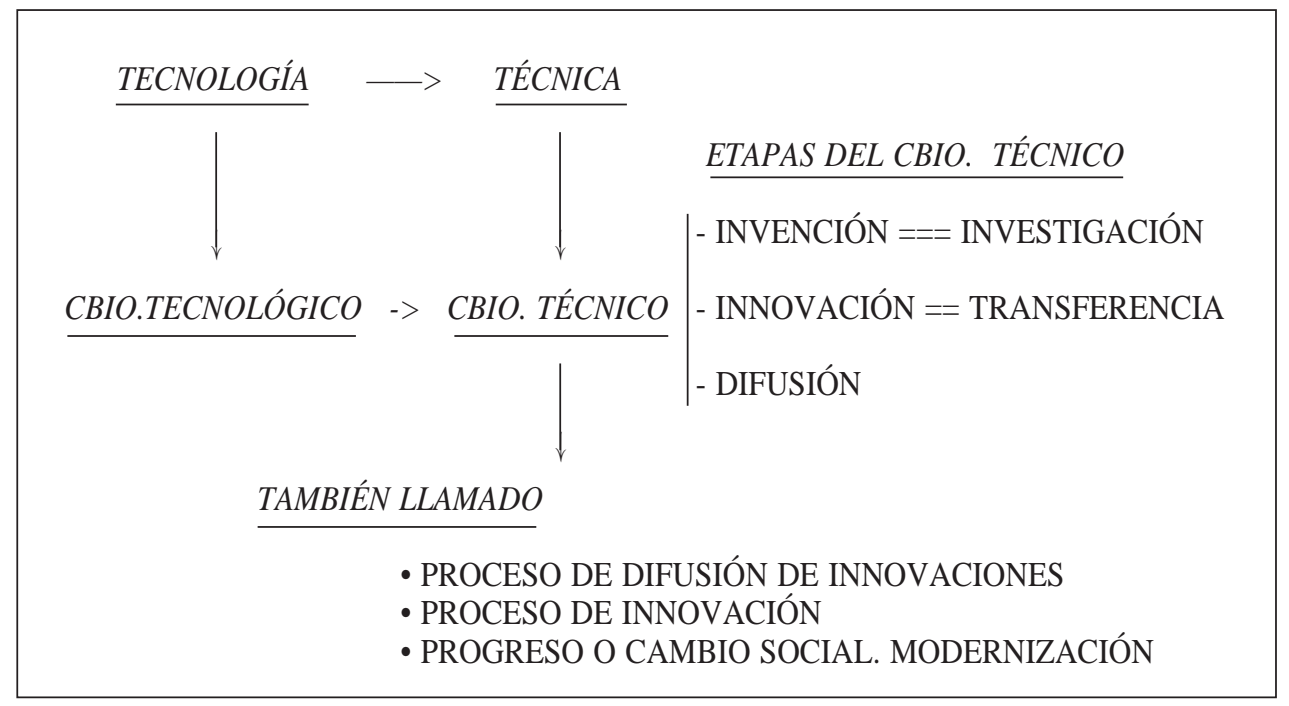

Dicho de otro modo, tradicionalmente los estudios sobre la difusión de innovaciones agrarias han tenido como elemento de análisis predilecto al agricultor, y en menor medida, en relación con las características personales de éste, a la innovación. Bajo este enfoque, de la adopción o de la demanda, la difusión es resultado de la corriente efectiva de información sobre la innovación y de las resistencias que ésta encuentra en los adoptadores.

Desde un enfoque más reciente, hecho desde la Geografía por Lawrence A. BROWN ${ }^{3}$, la adopción no sería más que la última de las tres etapas que darán lugar a la difusión. Las dos primeras se refieren a las acciones emprendidas por los propagadores, es decir, se centran en el lado de la oferta, complementario del anterior. Estas dos primeras etapas, que él llama de establecimiento de las agencias y de establecimiento y disposición de la innovación, van a ser decisivas para el posterior modelo de difusión resultante dado que en ellas se decide dónde, cuándo y cómo va a estar disponible la innovación.

3 Brown, L. A. (1981): «Innovation Diffusion. A New Perspective». 
LA DIFUSIÓN DEPENDE DE:

- CARACTERÍSTICAS DE LOS PROPAGADORES

- CARACTERÍSTICAS DE LOS ADOPTADORES

- CARACTERÍSTICAS DE LA INNOVACIÓN

LA DIFUSIÓN RESULTA DE LO ACONTECIDO EN LAS TRES ETAPAS DE:

- PUNTO DE VISTA DE LA OFERTA

1- ETAPA DE ESTABLECIMIENTO DE LAS AGENCIAS

2- ETAPA DE ESTABLECIMIENTO O DISPOSICIÓN DE LA INNOVACIÓN

- PUNTO DE VISTA DE LA DEMANDA

3- ETAPA DE ADOPCIÓN

Se trataba, pues, de reconstruir los tres estadios comprobando en qué medida se cumplían en el caso de la agricultura valenciana los supuestos que a nivel teórico se establecían en la bibliografía. Para ello, los elementos de análisis iban a ser tanto los propios agricultores (adoptadores), como también todos los segmentos que intervinieran en la promoción de la innovación entre aquéllos. A éstos se les clasificó en dos grupos:

-A) Oferta sin motivación de beneficios. En este grupo se situarían los servicios de la administración autonómica, los centros de investigación públicos, las universidades, las cooperativas y otras asociaciones de profesionales.

-B) Oferta con motivación de beneficios, entiéndase las firmas comerciales suministradoras de la innovación.

Se optó por estudiar la difusión de un conjunto de siete técnicas, todas ellas referidas al proceso de producción, que cuentan con redes de distribución y que dan en conjunto el tradicionalmente aceptado carácter moderno de la agricultura valenciana ${ }^{4}$.

Se eligió una metodología de tipo cualitativo, recogiendo la información necesaria a través de encuestas y entrevistas personales a elementos considerados representativos de los distintos grupos de estudio.

Desde el lado de la demanda, los entrevistados debían ofrecer una información de sí mismos, entendidos como sujetos de análisis, a la vez que una visión de conjunto del colectivo o segmento al cual representaban. Desde el lado de la oferta, sin motivación de beneficios, se analizó la actuación de los distintos estamentos que participan en las tres fases del proceso de difusión: la investigación, la transferencia y la difusión propiamente dicha.

4 Productos fitosanitarios, abonos, semillas híbridas o mejoradas, plásticos para la protección de cultivos, maquinaria, sistemas de riego localizado por goteo e invernaderos. 


\section{FASES DEL PROCESO DE DIFUSIÓN}

\section{- INVESTIGACIÓN}

- TRANSFERENCIA TECNOLÓGICA

Proceso mediante el cual los resultados de la investigación son introducidos en el sistema productivo.

INVENCIÓN $\longrightarrow$ TRANSFERENCIA $\longrightarrow$ INNOVACIÓN

FORMAS DE TRANSFERENCIA EN EL SECTOR AGRARIO

a) EXPERIMENTACIÓN

b) CAPACITACIÓN

FORMA DE TRANSFERENCIA ÓPTIMA

IMPLICACIÓN DE LA EMPRESA EN LAS ACTIVIDADES GENERADORAS DE LA INNOVACIÓN: INVESTIGACIÓN Y DESARROLLO.

$\underline{\text { DIFUSIÓN }}$

Para la oferta privada, o con motivación de beneficios, el análisis se centró en la última fase, la de difusión, en las actividades desarrolladas por las empresas suministradoras en las dos consabidas etapas de establecimiento de las agencias y de establecimiento o disposición de la innovación.

\section{Resultados}

\subsection{La difusión de innovaciones desde el lado de la oferta}

\subsection{A. La oferta sin motivación de beneficios}

1. La INVESTIGACIÓN es la actividad que aparece más claramente definida y organizada. El objetivo de la misma es asegurar la eficacia de la producción agraria, la competitividad del sector y las rentas de los agricultores mediante la reducción de costes. Esta reducción de gastos se basa en un uso más racional de los factores de producción.

Los problemas que se plantean en ella se centran fundamentalmente en su aspecto organizativo, incluida su evaluación, y en la conexión de sus actividades y resultados con los beneficiarios finales.

2. Mucho tiene que ver esta última cuestión con la fase de TRANSFERENCIA, aquélla que se ha revelado como la de mayores deficiencias.

Objetivo prioritario de la política tecnológica, la participación de la empresa agraria en las actividades de investigación y desarrollo es prácticamente nula. El motivo que explica tal circunstancia es que desgraciadamente no puede hablarse de empresa agraria; la explotación nunca ha dejado de serlo. 
Lejos de esta nueva concepción de la transferencia, en el sector agrario se ha identificado esta actividad con el mero acercamiento de los resultados de la investigación al agricultor. Este acercamiento de ha producido de dos formas:

- bien mediante la adaptación y simplificación de los resultados de la investigación a las condiciones reales de campo; a través de la experimentación,

- bien mediante el intento de elevar la formación del usuario de la técnica con el fin de facilitar su adopción; a través de la capacitación.

Dado lo inadecuado de esta forma de entender la transferencia, además de los defectos de funcionamiento que también presenta, se plantea la necesidad de cambiar la orientación del actual Servicio de Transferencia de Tecnología de forma que este cambio suponga una mayor vinculación de la «empresa agraria» en los procesos de investigación y desarrollo, defendiéndose el carácter externo a la explotación que estas actividades deberían tomar dada su especial naturaleza.

3. Las actividades de DIFUSIÓN desde la oferta sin motivación de beneficios, llevada a cabo por los servicios que realizan la extensión agraria, cuentan asímismo con graves defectos derivados de una mala estructura organizativa y una exigente política presupuestaria de minimización de costes.

A nivel organizativo cabe destacar el excesivo número y mal reparto de las agencias a lo largo del territorio valenciano. Esto se traduce en una mala dotación de medios tanto materiales como humanos en cada una de ellas, hecho que obliga al empleo de estrategias de difusión excesivamente homogéneas cualquiera que sea el tipo de técnica a difundir. Además, provoca el excesivo recurso a la comunicación en dos etapas, política selectiva de transmisión de la información que contribuye a generar mayor diferenciación entre los individuos de una misma comunidad rural.

Por lo tanto, se plantea como conveniente una reducción y concentración del número de agencias con mayores dotaciones y atribuciones competenciales que en la actualidad, y por una serie de criterios más de orden político-administrativo que de efectividad, detentan otros servicios, especialmente el Servicio de Transferencia de Tecnología Agraria. Es necesaria una reasignación de funciones en sentido ascendente dentro del continuo investigación-transferencia-difusión con el fin de que el apoyo al cambio técnico y a la innovación se corresponda con una agricultura competitiva y desarrollada y no meramente subvencionada.

\subsection{B.) La oferta con motivación de beneficios}

Especialmente relevante era en este caso, como se ha dicho, la tercera fase de difusión, más que las actividades previas de investigación y de desarrollo y experimentación.

Varias ideas conviene resaltar. Todo apunta a un modelo de difusión preferentemente de tipo jerárquico, por las siguientes razones:

- En cuanto al establecimiento de las agencias:

- Generalmente, las firmas que comercializan la innovación no desarrollan una nueva infraestructura de distribución propia sino que recurren a distribuidores preexistentes instalados, más probablemente, en los núcleos poblacionales más importantes 5 .

- La estructura organizativa predominante en estas redes es en consecuencia de tipo descentralizado, dependiendo la influencia del coordinador de propagación de la densidad de adoptadores, siendo aquélla más importante cuanto mayor sea ésta.

5 Teoría de Pedersen, P. O. (1970): «Innovation Diffusion within and between National Urban Systems». 
- Los criterios de localización o de contratación de las agencias por parte de las firmas suministradoras son ante todo la importancia del mercado potencial y la existencia de propagadores o distribuidores dispuestos a colaborar con ellas.

Otros factores económicos como los costes de establecimiento y mantenimiento de las agencias, los de distribución de la innovación o la proximidad al mercado, son menos importantes.

- En cuanto a las estrategias de difusión empleadas durante la etapa de establecimiento o disposición de la innovación:

Suelen ser siempre las mismas, excepto en el caso de las técnicas de mayor consumo y con un ritmo de renovación más importante.

- Aunque la innovación se hace disponible generalmente en todos los puntos de la red al mismo tiempo, en casos excepcionales de limitación en su disposición, circunstancia más probable en el período incial de su introducción, la innovación se dirige a localizaciones escogidas (por volumen de ventas, por importancia, proximidad o confianza del cliente, etc.). Todo ello refuerza la tendencia jerárquica de la difusión.

- La política de ventas predominante es la de encontrar un adecuado orden de rentabilidad entre costes y beneficios. Dependiendo de la etapa del ciclo de vida de la innovación, la política puede variar desde la maximización de ventas, en las etapas iniciales de introducción cuando las innovaciones todavía son jóvenes con lo cual se darían previsiblemente efectos de jerarquía, hasta la minimización de los costes, cuando se tratara de innovaciones ya maduras, en cuyo caso predominarían los efectos de vecindad en la difusión.

- Por otra parte, la información sobre la innovación no se hace al mismo tiempo para todos los individuos. Siempre existe una segmentación, explícita o implícita, del mercado. La comunicación en dos etapas vuelve a revelarse como una de las formas predilectas de transmitir la información, barata y rentable. Es una muestra de que los medios de comunicación interpersonales se prefieren y se reconocen de mayor impacto que los masivos; sin embargo esto no significa que exista un claro predominio en el uso de unos sobre los otros.

Así pues, puede deducirse que los efectos predominantes en la difusión son de tipo jerárquico, jerarquía que coincide con la del sistema urbano dada la naturaleza del mismo en el País Valenciano, para posteriormente darse los de vecindad. Entonces, la velocidad y el alcance de la difusión dependerá de las características personales del adoptador, de entre las que las locacionales no parecen ser especialmente significativas. Se correspondería este modelo con el establecido por HÄGERSTRAND para la difusión de las innovaciones de consumo a través de un sistema urbano ${ }^{6}$.

Ciertamente existen diferencias en la dotación de infraestructuras de suministro de unas áreas a otras, generalmente en favor del litoral, donde se encuentran las áreas de mayor consumo, por el tipo de agricultura practicada y por su mayor población, aspectos ambos interrelacionados. Sin embargo, las distancias que separan las agencias, por la forma y extensión del País Valenciano, no pueden considerarse como barrera o impedimento a la difusión.

\subsection{La difusión de innovaciones desde el lado de la demanda}

De mayor importancia que las locacionales son otras características individuales de los adoptadores. Cuatro son los objetivos que se pretenden cubrir en este apartado:

1) Saber cuáles son las variables personales con mayor repercusión en el proceso de adopción, así como los valores y actitudes predominantes ante el cambio: motivación, grado de satisfacción provocado y actitud futura ante él.

6 Hägerstrand, T. (1967): «Innovation diffusion as a spatial process». 
a) CARACTERÍSTICAS PERSONALES DEL ADOPTADOR:

- LOCACIONALES

- SOCIOECONÓMICOS

- PSICOLÓGICOS

— DE COMPORTAMIENTO SOCIAL

b) VARIABLES Y ACTITUDES

- MOTIVACIÓN AL CAMBIO

- CARACT. PERCIBIDAS DE LA INNOVACIÓN

- VALORES Y OBJETIVOS QUE INFLUYEN EN EL CAMBIO

- VENTAJAS PERCIBIDAS DE LA ADOPCIÓN DE LA INNOVACIÓN

— GRADO DE SATISFACCIÓN PROVOCADO POR EL CAMBIO - ACTITUD FUTURA ANTE EL CAMBIO

2) Cuáles son los principales mecanismos o procesos que llevan a la adopción, reconociendo el cumplimiento o no de las distintas fases, cinco, que se establecen a nivel teórico: conocimiento, interés, evaluación, prueba y adopción.

3) Cuáles son los principales estrangulamientos que limitan la adopción.

4) De qué formas se solucionan esos problemas.

1) Por lo que respecta a las variales personales positivamente relacionadas con el cambio, un buen acceso a los canales de información se demuestra como primordial para que se produzca la adopción, más que cualquier otra característica.

De entre ellas, que hemos agrupado en cuatro conjuntos, las correlaciones positivas y negativas coinciden con las hipótesis establecidas a nivel teórico. Características socioeconómicas como el tamaño de explotación, relacionado con la orientación productiva, el nivel de formación y la capacidad de gestión, relacionada a su vez con edades jóvenes, el nivel de educación y formación profesional agraria, por lo general muy bajo, se encuentran positivamente relacionadas con la adopción. La edad, especialmente en los segmentos superiores, por encima de los 55-60 años, lo está negativamente.

Características psicológicas como la propensión al riesgo, menos racional y decidida que resignada, al cambio, muy habitual aunque a veces no responde a una voluntad propia sino a la mera necesidad ${ }^{7}$, y al crédito, mucho menos común que las dos anteriores, se encuentran positivamente relacionadas con la adopción de la innovación. Mientras, el fatalismo, entendido como la propia incapacidad para poder decidir sobre su futuro, es una actitud negativa muy común entre el agricultor valenciano.

7 Idea del efecto noria o 'Technological Treadmill' de Cochrane, W.W. (1958): «Farm Prices, Myth and Reality». El agricultor se ve forzado a adoptar la técnica porque de no hacerlo queda apartado. Esta obligación supone un endeudamiento progresivo que no le es posible recuperar debido al precio cada vez mayor de los «inputs» necesarios, incremento que no tiene reflejo en los precios percibidos por la producción.

El símil de la noria sitúa al agricultor en uno de los peldaños de la misma en dirección descendente. Para poder mantenerse debe ir escalando peldaños sucesivamente, pero cuanta mayor velocidad imprime a su movimiento, la noria lo empuja más deprisa hacia abajo. 
Figura 1. Características percibidas de la innovación (precio)

FiguRA 2. Características percibidas de la innovación (complejidad)

También se consideraron algunas características de comportamiento social tales como el cosmopolitismo y participación social. Correlacionadas ambas positivamente con la adopción, la primera es mucho más frecuente dado el carácter abierto a influencias externas del agricultor valenciano; sin embargo, el nivel de participación social es mucho menos habitual.

Se produce de esta forma una alternancia entre características positivas y negativas para la adopción. Esta combinación da finalmente como resultado una difusión muy rápida en la que el componente imitativo es fundamental gracias a la velocidad con la que fluye la información y gracias a la permeabilidad que el agricultor presenta ante la misma.

Una profunda tradición agrícola, la disposición de unas consolidadas redes de distribución de la información y de la innovación que se ocupan de hacer llegar continuamente al 
Figura 3. Motivación del cambio. Valores que influyen en la decisión de adopción.

Figura 4. Principales valores económicos que influyen sobre la decisión de cambio.

sector las novedades técnicas dado que su receptividad lo convierte en un apetecible mercado, así como la relación entre las distintas innovaciones difundidas formando paquetes tecnológicos, explican la rapidez y facilidad del cambio.

Además de las características de los adoptadores, también las de la innovación tienen su influencia sobre la decisión de adopción. En este caso el precio se constituye como factor mucho más limitativo que la complejidad técnica. Esta o la escasa formación del adoptador no son obstáculo para que la adopción se produzca, aunque esto suponga la elección de una técnica inadecuada, que no se domina completamente y a la que no se le podrá sacar todo el rendimiento que por sus potencialidades sería posible.

En cuanto a los valores y actitudes que provocan el cambio, estos son clarísimamente de tipo económico: aumentar la producción, hacer la cosecha más segura y rentable, aumentar los beneficios y reducir los costes.

A enorme distancia figuran los valores intrínsecos (mejorar las condiciones de su actividad profesional) y a más aún los expresivos.

La impresión que el agricultor tiene de la innovación y de su adopción es generalmente positiva. Las mayores ventajas que dice encontrar en ella son generalmente, una vez más, 
FIGURA 5. Ventajas percibidas de la adopción de la innovación.

FIGURA 6. Ventajas económicas percibidas de la adopción de la innovación.

de tipo económico, especialmente la disminución de costes o el mayor rendimiento de la cosecha, lo que se traduce en un incremento de los beneficios.

El mismo componente económico puede presuponerse en la segunda de las respuestas más frecuente en esta misma cuestión: la obligatoriedad del cambio para poder mantenerse en la actividad. Ventajas personales como la comodidad u otras como las agronómicas quedan en un segundo plano.

El agricultor se encuentra por lo tanto satisfecho de la adopción, repartiéndose los motivos entre los económicos, traducidos en un incremento de rentas, y en segundo lugar los expresivos, donde la realización personal y el ser un buen profesional son los valores dominantes. Muy descolgados quedan los valores intrínsecos, reducidos únicamente a la valoración de la actividad agraria como ocupación deseada. 
FIGURA 7. Importancia de los valores causantes de la satisfacción de la adopción.

FIgURA 8. Tipos de valores causa de la satisfacción de adopción.

Por regla general se considera que a la innovación se le obtiene un rendimiento, en caso de que éste no sea evidente las causas no se deben a la propia innovación sino a la mala coyuntura del sector. Llama la atención la proximidad e incluso «afecto» a la innovación, cuya adopción debería responder a cuestiones de rentabilidad, ligadas obviamente a la marcha de los mercados agrarios, independientemente de si en sí misma es positiva o no.

Todas estas circunstancias hacen que la actitud futura ante el cambio sea positiva, siendo más comedida para aquellas innovaciones cuya adquisición supone un fuerte desembolso de capital. Posibilidad de financiación y obtención de rentabilidades son entonces las condiciones mayormente exigidas. 
FIGURA 9. Tipos de fuentes utilizadas en la etapa de conocimiento.

FIGURA 10. Tipos de medios interpersonales empleados en la etapa de conocimiento.

2) Mecanismos o etapas que llevan a la adopción.

Se dio por supuesta únicamente la existencia de dos de las cinco fases del proceso: conocimiento y adopción ${ }^{8}$. Se trataba de constatar las características que presentaba cada cuál y si se reconocían las tres intermedias.

La explotación de los resultados demostró que en la primera fase, la de conocimiento, los medios de comunicación interpersonales eran los mayormente utilizados, contrariamente a lo que a nivel teórico se establecía.

8 Primera y última según tipología reconocida por Rogers, E. M. (1962): «Diffusion of innovations». 
FIGURA 11. Fases intermedias del período de adopción.

FIGURA 12. Fases intermedias del período de adopción por tipo de innovación.

Generalmente la información sobre la innovación el adoptador la obtiene, por este orden, de otros colegas, de la oferta privada y de la coopertativa, constituyéndose el suministro como la fuente de información principal.

Estos resultados resaltan por un lado la importancia de la existencia de una infraestructura para la adopción, por otro la rapidez y efectividad con la que correrá la información y se hará posible la adopción.

De las tres fases intermedias reconocidas desde la teoría, la más importante de todas ellas es la de interés, traducida en la observación de lo que hacen otros, demostración en condiciones de campo por parte especialmente de otros agricultores aunque también de los propagadores. 
Figura 13. Causas de la decisión final de adopción según origen de la persuasión.

FIGURA 14. Causas de la decisión final de adopción: persuasión ajena.

La fase de prueba se reconoce en menos ocasiones, reduciéndose a las innovaciones divisibles que toleran mal un posible fracaso9.

Llama poderosamente la atención la poca importancia que cobra la etapa de evaluación. Puede traducirse en el hecho de que el adoptador es fácilmente convencido en la fase de interés, o incluso en el mismo tiempo del conocimiento, dándose en ocasiones, y en las innovaciones de menor coste, todas las fases en un mismo tiempo. La respuesta «no hay desfase entre conocimiento y adopción» cobra una especial significación gracias a ese tipo de técnicas ${ }^{10}$.

Las causas de la decisión final de adopción son claras:

- la demostración por parte de otros agricultores,

- la prueba en la explotación o

9 Semillas y productos fitosanitarios.

10 Productos fitosanitarios, abonos y plásticos para la protección de cultivos. 
FiguRA 15. Número de agricultores según tipos de problemas reconocidos en la explotación.

- la propia decisión personal, decisión que cuando se trata de técnicas más novedosas, específicas o técnicamente más complejas se sustituye por el consejo de los técnicos; cuando no la misma necesidad de poder mantenerse en la actividad.

Predomina, en cualquier caso, la persuasión ajena sobre la propia.

Todas estas circunstancias indican una vez más el fuerte carácter imitativo y guiado del cambio técnico en la agricultura valenciana, contando menos la propia decisión personal. La explicación hay que buscarla en la escasa cualificación y preparación del adoptador. En consecuencia, si sobre un eje de coordenadas se situara el número acumulado de adoptadores a lo largo del tiempo, sería de esperar un modelo de difusión en forma de curva sigmoidea pero con una gran dosis de verticalidad en la fase intermedia de la curva que correspondería a los imitadores.

3) Principales problemas para la adopción.

Se distingue claramente entre lo que son los problemas de la explotación, de mayor trascendencia para el cambio técnico en su conjunto, de lo que son los problemas propios en el mismo momento de la adopción de una técnica en particular.

De entre los problemas de la explotación sobresalen especialmente los comerciales, entiéndase la imposibilidad de colocar el producto en el mercado a unos buenos precios. En relación con el comercial aparece el problema económico, traducido en una progresiva pérdida del nivel de rentas y de disponibilidad de capital líquido.

En un segundo nivel se situarían los problemas estructurales y de formación o capacitación, cuya importancia, curiosamente, es menos evidente para el conjunto de adoptadores $^{11}$. Podrían añadirse a estos últimos los agronómicos, los técnicos (complejidad de la innovación) y los financieros (facilidad de acceso al capital de riesgo).

Al contrario que los problemas de la explotación, la importancia concedida a los problemas habidos en el mismo momento de la adopción es mucho menor, y la forma de solución mucho más clara, aunque las respuestas varían en sentido inverso según aumente el coste por unidad de producto o la frecuencia de uso sea menor.

11 Puede decirse que el agricultor reconoce bien los problemas, con un sentido economicista bastante evidente, pero es incapaz de reconocer las últimas causas. 
Las respuestas predominantes son las de no haber tenido problemas en el momento mismo de la adopción, y cuando los hay, éstos son principalmente de tipo económico, en concreto la escasa disponibilidad de capital líquido, los mismos que se citaron como limitación máxima para una futura adopción. En un segundo nivel se situaron los de tipo técnico, de deficiencias en el suministro, de formación y los relacionados con el riesgo. Llama la atención la desaparición en este momento del problema de tipo comercial, asimilado por el de tipo económico, en el cual desemboca.

No obstante, los problemas en la adopción toman otra dimensión cuando en lugar de considerar la adopción de la técnica como una decisión aislada, a ésta se la considera como un elemento más de la actividad empresarial, interrelacionado con otros cambios de tipo organizativo y de gestión ${ }^{12}$. La existencia de esta capacidad organizativa es la que nos traslada de una agricultura meramente comercial a otra desarrollada.

El tránsito de la actual situación de agricultura comercial a agricultura moderna o desarrollada es mucho más importante, cualitativamente, del que se está produciendo desde las áreas mediterráneas más meridionales que están reconvirtiendo una agricultura antigua de subsistencia en otra comercial gracias al aprovechamiento de sus recursos endógenos, tanto físicos como humanos (mano de obra), modificando la función de producción mediante la introducción de nuevas técnicas de no excesiva complejidad.

El cambio hacia una agricultura plenamente desarrollada, al que la agricultura valenciana debe tender y al que se ve obligada por la competencia de estos países, especialmente de los vecinos norteafricanos donde justamente se han instalado grandes firmas de origen valenciano, necesita de la combinación de una serie de elementos que influyen decisivamente en la capacidad empresarial del profesional agrario.

Estos elementos son al menos:

- el tamaño de la explotación, y su capacidad para convertirse en empresa, tránsito que depende en mucho de...

- la capacidad organizativa, entendida como capacidad de gestión de todo el conjunto de actividades a desarrollar dentro de la empresa, necesarias para poder obtener éxito;

- en relación con esta capacidad organizativa, la calidad del capital humano, traducida en un nivel de formación suficiente; y por último

- la tecnología, considerada no como un elemento exógeno e independiente de la empresa, que puede adquirirse puntualmente sin más esfuerzo que el desembolso económico, sino como parte integrante de su propia organización interna. La empresa debe participar en las tareas de investigación y desarrollo. Si esta participación no es, o no puede ser, individual, cada empresa por sí sola, sí puede ser mancomunada con otras a través de los organismos de investigación y transferencia tecnológica dispuestos a tal fin.

Es precisamente en esta combinación compleja de elementos donde aparecen las principales dificultades para el agricultor valenciano, que es permeable a la información de la innovación pero que no sabe o no puede articularla convenientemente.

Las principales deficiencias se encuentran en el nivel de formación y en las características estructurales de las explotaciones, deficiencias que acaban por desembocar en una tercera como es la dificultad de acceso a las técnicas más novedosas y competitivas del momento, las de nivel superior, cuya oferta no se considera interesante o rentable por parte de sus propagadores.

La incapacidad de gestión a la que se está haciendo referencia, evidentemente ligada a su cualificación, es patente cuando entramos en el cuarto de los objetivos, el de reconocer las distintas formas de solución planteadas a las dificultades existentes.

12 Entronca este comentario con el de la nota inmediatamente anterior. 
4) Las soluciones o las ideas de solución son mucho más claras si los problemas se refieren al momento mismo de adopción que si lo hacen al conjunto de la explotación.

El agricultor parece ser capaz de reconocer y afrontar los problemas en un nivel inmediato o próximo porque se encuentra familiarizado con todas las variables que le están afectando, escasas y bien conocidas. Las soluciones se plantean entonces de forma personal. Sin embargo, es incapaz de realizar abstracciones o reflexiones más amplias para las que es necesario relacionar una serie de variables sentidas como exógenas. Hay una serie de problemas que por proximidad se reconocen como propios, a los cuales se les intenta dar solución, pero hay otros a los que no se sabe cómo hacerles frente por considerar que no tienen una componente individual sino colectiva o sectorial.

De este modo, se cree que los problemas de la explotación tienen una solución difícil y quien puede dársela pocas veces es el propio individuo, recayendo entonces toda la responsabilidad en la «socorrida» administración, lo cual ilustra bien a las claras el claro déficit competitivo de muchos representantes de este sector productivo.

El desentendimiento de los problemas de la explotación por parte del individuo todavía se hace más patente cuando se le pregunta la forma de darles solución. Si no es a través de un mayor apoyo y defensa institucional de la agricultura, no se sabe cómo. Únicamente la asociación de profesionales para resolver los problemas de tipo comercial, opción sin embargo que debe tomarse más como fórmula defensiva que como estrategia de progreso, y la iniciativa personal de unos pocos para resolver deficiencias en la formación individual, parecen salirse de la tónica general.

\section{Conclusión}

En síntesis, existe una importante incapacidad organizativa que se traduce en la imposibilidad de provocar innovaciones radicales, de producto, que permitieran a la agricultura valenciana encontrar sin excesivas presiones de la competencia un adecuado nicho en el mercado internacional. Los negativos efectos de esta incapacidad superan los positivos que se derivan de la actitud proclive o favorable a la introducción de técnicas de proceso. Dada la creciente competencia a la que se ve sometida la agricultura valenciana esto supone la pérdida progresiva de ese margen ventajoso del que solía disfrutar hasta ahora.

Dentro del aceptado carácter favorable a la modernización, las diferentes respuestas del individuo ante el cambio se explican por la distinta relación que los adoptadores tienen con la información (o con las fuentes que la suministran, a ella o a la misma innovación), relación que a su vez depende de sus propias características personales.

Así pues, puede decirse que la agricultura valenciana se caracteriza por una permeabilidad a las innovaciones necesarias para el proceso de producción, pero queda claramente en suspenso una mejora en los niveles superiores de capacidad organizativa y de gestión, claramente vinculados con la principal problemática del control de los mercados y de la competencia. Esta caracterización no es sino fiel reflejo del nivel de desarrollo en el que actualmente permanece, típico de las agriculturas comerciales, escalón previo, y distante, como se decía, de las agriculturas desarrolladas o modernas.

Parece evidente, pues, por todo lo expuesto, que la necesidad de cambio estriba prioritariamente en los niveles organizativos y de gestión de la actividad (explotación, versus, empresa). Es en ellos donde se encuentran las principales dificultades. El cambio técnico se interpreta precisamente como fruto o consecuencia de esa capacidad. Es esta potestad organizativa condición necesaria para poder desarrollar un nuevo papel de la empresa agraria en el proceso de creación de la innovación merced a una mayor participación de la misma en los procesos de investigación y desarrollo. Cabe insistir una vez más en que esta 
participación se entiende, dada la indiscutible particularidad del sector y de la empresa agraria, de una forma 'externalizada', en conjunción con los organismos e instituciones públicos y privados para los que también se requiere de la modificación necesaria que les acerque más a las explotaciones.

Cualquier otro intento de solución que se planteara para los problemas técnicos o comerciales se interpreta como un parche, pero no como una solución integral, que perpetuaría la actual situación de dependencia del agricultor respecto de estamentos y sectores externos, bien sea en los problemas técnicos, bien en los problemas comerciales.

Para que este cambio se produzca es necesaria, en gran medida, la mejora de la cualificación y preparación del agricultor, siendo conveniente una homogeneización del diseño curricular con respecto a cualesquiera otros profesionales, evitando formas de cualificación «particulares».

Finalmente, señalar también la necesidad de obtener las suficientes economías de escala, que no tienen por qué lograrse únicamente con un aumento del tamaño de la explotación. Las vías asociativas y la externalización de la propia empresa de estas actividades en el sentido expuesto, pueden ser alternativas completamente válidas.

Es posible que planteamientos como los que se acaban de hacer resulten de difícil asimilación o se entiendan como excesivamente alejados de las condiciones reales que presentan la mayor parte de las explotaciones y de los agricultores de la Comunidad; sin embargo, las posibilidades del sector, que apuntan únicamente en esa dirección, y también la afortunada existencia de algunos empresarios que por iniciativa individual empiezan a escrutar este camino, no vienen sino a confirmarlos.

\section{Bibliografía}

APARICI, A.; ABELLA, M. y MARTI, A. (1990): «Caracterización Sociológica del Agricultor Valenciano», informe mecanografiado realizado por encargo del Servicio de Estudios Agrarios y Comunitarios, Conselleria d'Agricultura i Pesca.

ARNALTE ALEGRE, E. (1980): «La agricultura a tiempo parcial en el País Valenciano», Madrid, Ministerio de Agricultura, Pesca y Alimentación.

ARNALTE ALEGRE, E. (1988): «Dinámica de una estructura agraria valenciana y lógica de una política de estructuras autónoma», 'Jornades de Política d'Estructures Agràries', Generalitat Valenciana, Conselleria d'Agricultura i Pesca, 1988, pp. 135-158.

ARNALTE ALEGRE, E. (1989): «Estructura de las explotaciones agrarias y externalización del proceso productivo», 'Información Comercial Española', Febr., pp. 101-117.

AZNAR, J. (1989): «Cultura empresarial, organización y medios necesarios. La necesidad de profesionalizar al sector», 'Phytoma', no 5 , pp. 11-14.

BARCELÓ VILA, L. V. y GARCÍA ÁLVAREZ COQUE, J. M. (1986): «Repercusiones sobre la Comunidad Valenciana de la adopción de la política agraria comunitaria», 'Papeles de Economía Española', Serie 'Economía de las Comunidades Autónomas', Comunidad Valenciana, pp. 199221.

BENELBAS, L. (1982): «Economía de la intensificación agraria. La localización y el tamaño de la explotación como condicionantes», 'Información Comercial Española', nº 581, pp. 27-36.

BONONI, A. (1987): «La innovación, objetivo clave de la estrategia de desarrollo. El papel del T.A. (Technology Assesment) en las regiones», 'Presentación del programa FAST. Programa Europeo de Prospectiva y Evaluación de la Ciencia y la Tecnología', Presidencia de la Generalitat Valenciana, 1987, mecanografiado, $19 \mathrm{pp}$.

BROWN, L. A. (1975): «The Market and infraestructure context of adoption: A spatial perspective on the diffusion of innovation», 'Economic Geography', Vol. 51, n 3, pp. 185-216.

BROWN, L. A. (1981): 'Innovation Diffusion, a new perspective», London \& New York, Methuen, $345 \mathrm{pp}$. 
BROWN, L. A. \& COX, K. R. (1971): «Empirical Regularities in the Diffusion of Innovation», 'Annals of the Association of American Geographers', $\mathrm{n}^{\circ}$ 61, pp. 551-559.

BROWN, L. A.; MALECKI, E.J. \& SPECTOR, A. N. (1976): «Adopter Categories in a Spatial Context: Alternative Explanations for an Empirical Regularity», 'Rural Sociology', Vol. 41, $\mathrm{n}^{\circ}$ 1, pp. 99-118.

BROWN, M. A. (1980): «Attitudes and social categories: complementary explanations of innovation-adoption behavior», 'Environement and Planning', Serie A, Vol. 12, nº 2, pp. 175-186.

BROWN, M. A. (1981): «Behavorial approaches to the geographic study of innovation diffusion: problems and prospects», en: Kevin R. Cox \& Reginald G. Golledge (Ed.), 'Behavioral Problems in Geography Revisited', Methuen \& Co., New York, pp. 123-144.

BROWN, M. A. (1981): «Spatial Diffusion Aspects of Marketing Strategies», 'The Review of Regional Studies', Vol. 11, n 2, pp. 54-72.

BURRIEL DE ORUETA, E. L. (1971): «La Huerta de Valencia, sector sur. Estudio de Geografía Agraria», Valencia, Alfonso el Magnánimo y Caja de Ahorros de Valencia.

BUSCH, L.; BONANO, A. y LACY, W. B. (1989): «Ciencia, tecnología y reestructuración de la agricultura», 'Agricultura y Sociedad', nº 53, pp. 73-103.

CABALLERO VILLAR, P. y DE MIGUEL GÓMEZ, M. D. (1985): «La difícil evolución de las empresas agrarias familiares hacia una dimensión económica viable. Un estudio en la región valenciana», XIX Congreso Internacional de Economistas Agrarios, Banco de Crédito Agrícola, pp. 37-41.

CARTER, R. A. (1988): «Innovation in Urban Systems: The Interrelationships between Urban and National Economic Development», 'Annals of Regional Science', Vol. 22, nº 3, pp. 66-79.

COCHRANE, W.W. (1958): «Farm Prices, Myth and Reality», Minneapolis, University of Minnesota Press.

CONSELLERIA DE AGRICULTURA Y PESCA (1986): «Instituto Valenciano de Investigaciones Agrarias. Memoria de actividades 1984-1985».

CONSELLERIA DE AGRICULTURA Y PESCA (1987): «Memoria 1986».

CONSELLERIA DE AGRICULTURA Y PESCA (1987): «Memòria d'activitats del Servei d'Extensió Agrària. Any 1986».

CONSELLERIA DE AGRICULTURA Y PESCA (1988): «Memòria d'activitats del Servei d'Extensió Agrària. Any 1987».

CONSELLERIA DE AGRICULTURA Y PESCA (1988): «Memoria 1987».

CONSELLERIA DE AGRICULTURA Y PESCA (1989): «Memoria 1988».

CONSELLERIA DE AGRICULTURA Y PESCA (1990): «Memoria 1989».

COSCOLLA, R. (1985): «Seguridad en el uso de plaguicidas», 'Agricultura y Cooperación', nº 28, pp. 29-30.

FARINOS, J. (1988): «Difusión de tecnología, capacitación y extensión agraria en España y en Europa. La cuestión de las nuevas tecnologías y su repercusión en la agricultura», Madrid, Secretaría General Técncia del M.A.P.A., 203 pp.

FARINOS, J. (1988): «El proceso de innovación y difusión tecnológica con especial referencia a la agricultura», tesis de licenciatura, $438 \mathrm{pp}$. (Mecanografiado).

FARINOS, J. (1989): «El papel de la innovación en la agricultura. La dimensión espacial de la difusión de innovaciones como factor de desarrollo', 'Cuadernos de Geografía', no 46, pp. 149168.

FARINOS, J. (1992): «El imperativo del cambio o/y mejora tecnológica de la agricultura valenciana. Análisis de los factores limitativos en los procesos de innovación y difusión tecnológica y su repercusión sobre el desarrollo», Tesis doctoral, 828 pp., mecanografiado.

FERNÁNDEZ, A.; RODRÍGUEZ, L.; PAREJO, J. y GARCÍA, L. (1983): «Progreso Tecnológico y Agricultura», Madrid, Banco de Crédito Agrícola, 435 pp.

GARCÍA DELGADO, J.L. (1976): «A propósito de 'La agricultura en el desarrollo capitalista español'», en 'La cuestión agraria en la España contemporánea', Ed. José Luis García Delgado, pp. 525-553.

GARCÍA FERRANDO, M. (1976): «La innovación tecnológica y su difusión en la agricultura», Madrid, Secretaría General Técnica del M.A.P.A., 300 pp. 
GASSON, R.M. (1973): «Goals and Values of Farmers», ‘Journal of Agricultural Economics', n’ 24, pp. 521-542.

GENERALITAT VALENCIANA, CONSELLERIA D’AGRICULTURA I PESCA (1986): «El sector agrario valenciano», $31 \mathrm{pp}$.

GENERALITAT VALENCIANA, CONSELLERIA D'ECONOMIA I HISENDA (1984): «Plan Económico Valenciano 1. (1984-1987)».

GENERALITAT VALENCIANA, CONSELLERIA D'ECONOMIA I HISENDA (1987): «Plan Económico Valenciano 2. (1987-1991)».

GENERALITAT VALENCIANA, CONSELLERIA D'ECONOMIA I HISENDA (1991): «Programa operativo de la Comunidad Valenciana (90-93)».

GÓMEZ MUÑOZ, A. C. (1986): «Difusión-Adopción de innovaciones en agricultura: Un estudio sobre la campiña de Córdoba», Tesis Doctoral.

GÓMEZ MUÑOZ, A. C. (1990): «Análisis socioeconómico de la innovación agraria», 'Curso Internacional de Estrategia y Planificación Alimentaria', mecanografiado, 26 pp.

HÄGERSTRAND, T. (1952): «The propagation of innovation waves», Lund, Lund Studies in Geography.

HÄGERSTRAND, T. (1967): «Innovation diffusion as a spatial process», Chicago, University of Chicago Press.

HOLT, J. E. (1985): «Technological change in agriculture. The system movement and power», 'A gricultural Administration', nº 18, pp. 69-80.

HONRUBIA LÓPEZ, J. (1988): «Caracterización de la agricultura valenciana», 'Revista Valenciana d'Estudis Autonòmics', no 11-12, pp. 171-181.

ILBERY, B. W. (1977): «Point score analysis: a methodological framework for analysing the decision-making process in agriculture», 'Tijdschrift voor economische en sociale geografie' ('Journal of economic and social geography'), Vol. 68, n' 2, pp. 66-71.

ILBERY, B. W. (1983): «Goals and Values of Hop Farmers», 'Translations of the Institute of British Geographers', no 8, pp. 329-341.

ILBERY, B. W. (1984): «Agricultural Specialization and Farmer Decision Behaviour: A case of Study of Hop Farming in the West Midlands», 'Tijdschrift voor economische en sociale geografie', Vol. 75, no 5, pp. 329-334.

JORDÁN GALDUF, J. M. (1986): «Agricultura y modernización. Algunas reflexiones sobre el caso valenciano», 'Revista Valenciana d'Estudis Autonòmics', no 5-6, pp. 107-122.

JORDÁN GALDUF, J. M. (1989): «España frente a los terceros países mediterráneos», Valencia, Generalitat Valenciana, $114 \mathrm{pp}$.

LÓPEZ ESTORNELL, M. (1985): «Algunos aspectos de futuro para la agricultura valenciana en relación con la CEE», 'Revista Valenciana d'Estudis Autonòmics', nº 3, pp. 145-149.

LÓPEZ ESTORNELL, M. (1986): «La agricultura valenciana y la crisis económica», 'Papeles de Economía Española', Serie 'Economía de las Comunidades Autónomas', Comunidad Valenciana, pp. 187-198.

MAROTO I BORREGO, J. V. et Alt. (1989): «Aproximación a un análisis descriptivo de los sistemas de producción agrarios en las comarcas valencianas», Valencia, Generalitat Valenciana, Conselleria d'Agricultura i Pesca, 794 pp.

MAROTO I BORREGO, J. V. et Alt. (1989): «Diagnóstico tecnológico de los sectores productivos de la agricultura valenciana», Valencia, Conselleria d'Agricultura i Pesca, 416 pp.

MARRON GAITE, M. J. (1989): «Los procesos de cambio en la agricultura. Factores que condicionan la conducta innovadora del agricultor», 'Estudios Geográficos', nº 194, pp. 91-110.

NAREDO, J. M.; LEAL, J. L.; LEGUINA, J. Y TARRAFETA, L. (1975): «La agricultura en el desarrollo capitalista español (1940-1970)», Madrid, Siglo XXI.

PERPIÑÁ GRAU, R. (1972): «De Economía Hispana», Barcelona.

PEDERSEN, P.O. (1970): «Innovation Diffusion within and between National Urban Systems», 'Geographical Analysis', Vol 2, nº 3, pp. 203-254.

PIQUERAS HABA, J. (1985): «La agricultura valenciana y su formación histórica», Madrid, M.A.P.A., $249 \mathrm{pp}$. 
PRED, A.R. (1975): «Diffusion, organizational spatial structure, and city system development», 'Economic Geography', Vol. 51, nº 3, pp. 252-258.

ROGERS, E.M. (1962): «Diffusion of innovations», New York, The Free Press.

ROGERS, E.M. \& SHOEMAKER, F.F. (1971): «Communication of innovations: A cross cultural approach», New York, The Free Press.

RÖLING, N. (1988): «Extension Science. Information Systems in Agricultural Development», Cambridge, University Press, $233 \mathrm{pp}$.

ROMERO GONZÁLEZ, J. (1989): «La agricultura valenciana en el proceso de industrialización y urbanización», Valencia, Conselleria d'Agricultura i Pesca, Generalitat Valenciana, 258 pp.

SPECTOR, A.N.; BROWN, L.A. \& MALECKI, E.J. (1976): «Acquaintance Circles and Comunication: An Exploration of Hipothesis Relating to Innovation Adoption», 'The Professional Geographer', Vol. 28, n ${ }^{\circ} 3$, pp. 267-276.

UNIVERSIDAD DE VALENCIA-ESTUDIO GENERAL. FUNDACIÓN UNIVERSIDAD-EMPRESA. A.D.E.I.T. (1991): «Informe de actividades».

UNIVERSIDAD DE VALENCIA-ESTUDIO GENERAL. OFICINA DE TRANSFERENCIA DE LOS RESULTADOS DE LA INVESTIGACIÓN (1991): «Informe de actividades».

UNIVERSIDAD POLITÉCNICA DE VALENCIA. CENTRO DE TRANSFERENCIA DE TECNOLOGÍA (1991): «Informe de actividades». 